\title{
Perceived Multimedia Quality: the Impact of Device Characteristics
}

\author{
Gheorghita Ghinea and Kyle J. Patterson \\ Brunel University, Uxbridge, United Kingdom \\ george.ghinea@brunel.ac.uk; dremzo@gmail.com
}

\begin{abstract}
In the study reported in this paper, the research aims to tackle the question "Do the hardware characteristics of a device affect the viewer's perceived quality of the media? Using different hardware devices and media clips that have had different frame rates the viewer's information assimilation and satisfaction of the media clip was measured. The results suggest that there is a deeper link between the user's information assimilation than just the hardware that the media is viewed on. On the other hand a user's satisfaction with clip quality is affected by the alterations in frame rate of the media rather than the device as the satisfaction levels increased regardless of the hardware characteristics.
\end{abstract}

Keywords: device, frame rate, perceived multimedia quality.

\section{Introduction}

With the ever increasing availability of media content and technological advances in visual displays, there is a general expectation that the media quality is increasing. As an example, media/video clips that are run at a higher frame rate, from a technical point of view, have higher quality because everything appears smoother to the eye of the beholder, the brain not being able to distinguish individual media frames. With higher frame rates come very large bandwidth and processing power requirements, which may be perfectly fine for video conferencing in a building with optical broadband connections and powerful videoconferencing units, but not a handheld mobile device.

Another area key to perceived media quality is the screen resolution of the displaying device. High Definition technology with display resolutions of up to 1920x1080 progressive suggesting 'a better viewing experience' is already wellestablished, with the next step - Quad High Definition - being able to run 3840x2160 progressive.

Although work has been undertaken to assess perceived/subjective media quality in terms of frame rate [1][3], resolution [2], and on specific devices [5][6], to the best of our knowledge, the question of how subjective media quality is impacted when the same content is viewed across different devices has not been explored. 


\section{Methodology}

\subsection{Subjects}

96 volunteers, aged between 16-38, participated in the experiment. Participation was voluntary, with all subjects being recruited from the staff and student population at Brunel University.

\subsection{Hardware Devices}

The four devices (Fig. 1) used to run the media in the experiment were a high definition television (22" LCD screen, 16:9 aspect ratio, 1920x1080 resolution), a notebook computer (15.6" LCD screen, 16:9 aspect ratio, 1440x900 resolution), a netbook (10.1" LCD screen, 16:9 aspect ratio, 1024x600 resolution) and a smart phone (3.5" TFT screen, 16:9 aspect ratio, 640x360 resolution).
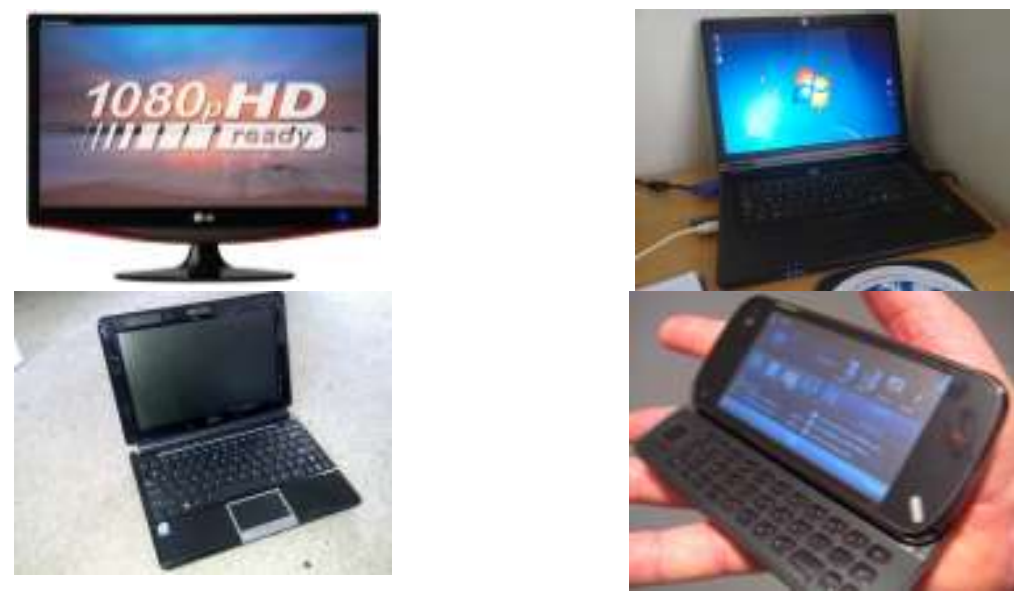

Fig. 1 Devices used in our study

\subsection{Media Clips}

12 video clips, with varying spatial and temporal characteristics, previously employed in perceptual quality studies (e.g. [4]) were used in our experiment. Each video clip was shown at one of three different frame rates: 5,15 , and 25 frames per second.

\subsection{Evaluation Metric}

The Quality of Perception (QoP) metric, assessing subjective media infotainment quality [4] was used in the experiment. QoP has two components, QoP-IA information assimilation representing the percentage of informational content assimilated by the end user, and QoP-S, the subjective media quality, expressed on a scale of 1-5. 


\subsection{Experimental Design}

Each participant was randomly assigned to view media on one of the four hardware devices, on which s/he viewed the 12 clips (4 clips were viewed at 5, 15 and $25 \mathrm{fps}$, respectively). In order to prevent order effects, the presentation order of clips was randomised. After watching each clip, the participant filled in the QoP questionnaire and then went on to view the next clip. Media on a particular device was viewed by a total of 24 participants.

Each participant was lead to a closed off room where the device was positioned on a table. The device was not moved through each participant however the addition of an office chair with wheels attached to the legs allowed for the participant to adjust themselves relative to how they felt comfortable watching the device. The device remained on the playlist screen whilst the participant was reviewing instructions about how the following experiment would unfold.

The participant was told they were about to watch 12 media clips and that after each clip they would be given a questionnaire to answer with questions relating to the media clip they had just seen. Participants were told that the questions will relate to the media clip and that no trick question would be asked.

\section{Results}

Analysis of Variance of the results suggest that there is a deeper link between the user's information assimilation than just the hardware that the media is viewed on. On the other hand a user's satisfaction with clip quality is affected by the alterations in frame rate of the media rather than the device as the satisfaction levels increased regardless of the hardware characteristics. However, the interaction between media content and the device on which it was played was shown to have a statistically significant impact on user subjective satisfaction with the media quality. Last but not least, irrespective of the device, our results suggest that there is a strong relationship between media content and the frame rate at which it is viewed.

\section{Future Work}

Our study has two possibilities for future work:

- The broadening of the device spectrum: with 60 " televisions existing in today's market incorporating devices from 60" displays to 3" displays would mean the results can be generalised across the population.

- Updating the experiment material with high definition resolutions for the media clips: the current media clips only running at $640 \times 480$ pixels meant that when shown on the larger screens the clips slightly distorted and may have influenced the results. 


\section{References}

1. Apteker, R.T., Fisher, J.A., Kisimov, V.S. and Neishlos, H. Video Acceptability and Frame Rate, IEEE Multimedia, 2(3), 32-40 (1995).

2. Bracken, C.C.: Presence and Image Quality: The Case of High-Definition Television, Media Psychology, (7)2, $191-205$, (2005).

3. Ghinea, G. Thomas, J.P.: QoS Impact on User Perception and Understanding of Multimedia Video Clips, Proceedings of ACM Multimedia '98, pp. 49-54 (1998).

4. Ghinea, G. Thomas, J.P.: Quality of Perception: User Quality of Service in Multimedia Presentations, IEEE Transactions on Multimedia, 7(4), 786-789 (2005).

5. Jumisko-Pyykkö, S. Häkkinen, J.: Evaluation of Subjective Video Quality of Mobile Devices, Proceedings of the 13th annual ACM international conference on Multimedia, pp $535-538$ (2005).

6. Kaasinen, E., Kulju, M., Kivinen, T., and Oksman, V.: User acceptance of mobile TV services. In Proceedings of the $11^{\text {th }}$ international Conference on Human-Computer interaction with Mobile Devices and Services, Bonn, Germany (2009). 